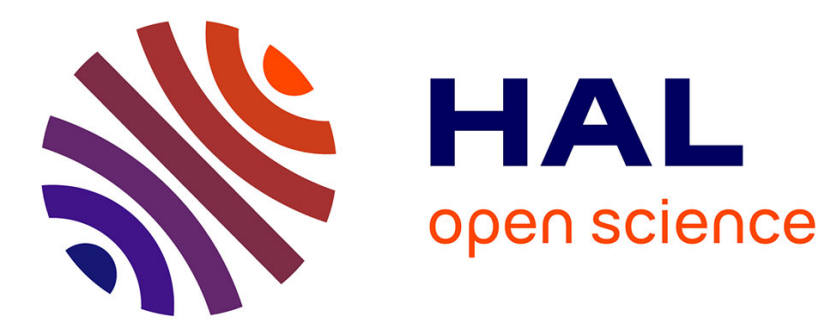

\title{
Asymmetric Awareness and Heterogeneous Agents
} Antoine Dubus

\section{To cite this version:}

Antoine Dubus. Asymmetric Awareness and Heterogeneous Agents. 2017. hal-01521487v3

\section{HAL Id: hal-01521487 \\ https://hal.science/hal-01521487v3}

Preprint submitted on 29 Jun 2017

HAL is a multi-disciplinary open access archive for the deposit and dissemination of scientific research documents, whether they are published or not. The documents may come from teaching and research institutions in France or abroad, or from public or private research centers.
L'archive ouverte pluridisciplinaire HAL, est destinée au dépôt et à la diffusion de documents scientifiques de niveau recherche, publiés ou non, émanant des établissements d'enseignement et de recherche français ou étrangers, des laboratoires publics ou privés. 


\title{
Asymmetric Awareness and Heterogeneous Agents
}

\author{
Antoine Dubus*
}

June 29, 2017

\begin{abstract}
We consider the principal-agent model with asymmetric awareness and introduce heterogeneity of the agents on their aversion to effort. We discuss the optimal contract and market structure in a market with an aware principal and unaware agents. When the principal faces two types of agents, one being more effort-averse than the other, the contract he proposes either pools them, separates them, or excludes the more effort-averse agents of the market depending on their proportion. When efforts are observable, all the agents remain unaware. When efforts are hidden the principal increases the awareness of the agents, to a level which depends on the nature of the contract.
\end{abstract}

口

Keywords: Economics of Contract: Theory; Unawareness; Asymmetric and Private Information; Insurance

JEL Classification: D82; D83; D86; G22.

\section{Preliminary version.}

*Telecom ParisTech (Economics and Social Sciences), 46 rue Barrault, 75634 Paris Cedex 13; Email: antoine.dubus@telecom-paristech.fr.

I am grateful to Yann Balgobin, David Bounie, Martin Quinn, Patrick Waelbroeck, the participants of the Digital Economics Seminar at Paris-Sud University and of the International Congress of the French Economic Association for helpful comments and suggestions. 


\section{Introduction}

The traditional agency problem with hidden efforts and moral hazard assumes that the principal and the agent have the same knowledge over the contingencies affected by the agent's efforts. However, as Auster (2013) shows, asymmetric awareness can be used by one of the contracting party to design the contract. Until now, models including awareness asymmetry have not introduced market refinements such as heterogeneity over the cost to effort of the agents. In this paper this heterogeneity is introduced into a model of contract with unawareness. Namely, the agents can be of two types, one being more effort-averse than the other. The main question of our paper is to know how this heterogeneity affects the design of the contract, especially in terms of level of awareness revealed to the agents. In an extension, the equilibrium resulting from competition is also studied, and the associated level of awareness disclosed by the principal.

Consider contracting in the health-insurance market. The current contracts stipulate a compensation based on an outcome that is the health of the agent. The insurance company that proposes the contract is probably more aware of what behavior affects the health of its clients than they do themselves. It designs the contract using this higher awareness as a way to maximize its profits. Assume that exerting a lifestyle effort increases the chances of the clients not to have a disease, for instance walking a certain distance each day reduces the chances of having a heart attack. Assume also that the clients are of two types, one very averse to effort and the other less. The question answered here is whether the insurance company reveals to the agents that they should walk a certain distance every day, and if it contracts with agents of both types. Indeed, the tradeoff emphasized by Auster (2013) is that revealing this contingency increases the agent's incentive to adopt a healthier behavior, but it also tightens the participation constraint.

Allowing for heterogeneous agents has strong implications for market equilibrium compared to the initial model. Namely, partially covered markets are allowed, thus, questions of market structure arise. Will the principal deal with one type of agents only, with both of them identically or with each of them differently? It is shown that it is not 
always optimal for the principal to offer a unique contract, but that depending on the proportions of high and low-type agents, he will either propose a contract fitting with the more effort-averse agent, or one offering two separate contracts for the two agents, or even one fitting with the less effort-averse agent, and implicitly excluding the other type from the market. In the second case, the more effort-averse agent is motivated to exert a low-level effort while the other type is encouraged to exert a high effort. The degree to which the awareness of the agents is enlarged depends on the type of contract chosen.

The model of agency with unawareness used here is developed initially by Auster (2013), we extend it to the case of agents with heterogeneous effort-costs. A risk-neutral principal proposes a contract to a risk-averse agent over the uncertain outcomes of a task. More precisely, the outcome is a function of the stochastic realization of binary contingencies. Awareness-asymmetry arises over the knowledge of the contingencies, since the principal knows the whole set of contingencies and the agent only part of it. The agents exert hidden efforts that affect the realization of the contingencies. The effort can be of two types, high or low, and the different effort-aversions of the agents come from a higher effort-cost for the low-type agent than for the high-type one.

The consequence of the asymmetric awareness is that the agents maximize their utilities using the probabilities of occurrence of each outcome as they perceive it. Auster (2013) shows first that the agents' unawareness implies that they do not contract on the outcomes they are not aware of, and do not get a retribution for it. She also shows that, in contracts with hidden efforts, the principal reveals some contingencies to the agents in order to increase their motivation to exert an effort, meaning that more awareness relaxes the incentive-compatibility constraint; but it also results in tightening the participation constraint of the agents. They ask for more retribution since they know more precisely the impacts of their effort.

In this set-up, it is shown first that the main results in Auster (2013) hold for the case with heterogeneous agents. In contracts with observable efforts, awareness is always minimal whatever the regime chosen by the principal. When efforts are hidden however, 
the agents' awareness is increased (but not always complete). Awareness in the different contracts may be higher or lower depending on the awareness structure and of the valuation consumers have over the compensation. We provide here the rules driving different degrees of awareness disclosure depending on the contracts. Also, we derive some sufficient conditions for the principal's strategy to change when skipping from a contract with hidden to observable effort for identical rates of high effort-cost agents in the population. For some agent-types proportions that in contracts with observable efforts imply a separating contract, skipping to observable efforts implies that the contract becomes either pooling or separating (depending on the proportion).

A brief review of the literature is provided in section 2. Section 3 is devoted to the description of the model of contract with unawareness. In section 4 the solution of the observable contract is outlined, while section 5 gives the results for the hidden effort case. Section 6 is devoted to an extension to a market in duopoly, and section 7 proposes a discussion over the market structures in equilibrium.

\section{A review of the literature}

The classical economic theory assumes that the economic agents have the same awareness on the factors affecting an outcome (see Holmström (1979) and Grossman and Hart (1983)). Maskin and Tirole (1999) put this symmetric-awareness assumption into question by explaining that "contractual incompleteness" occurs, and affects the structures of markets and even political systems. The aim of their paper is to show the irrelevance of transaction costs as a way to model why agents cannot apprehend contingencies ex ante. In particular, they emphasize the lack of a theory that allows for bounded rationality in the agency problem.

The literature on awareness aims at filling this gap. Starting with the seminal works by Modica and Rustichini (1994) and Dekel and al. (1998) papers like Heifetz et al. (2006) or Galanis (2011) propose models that allow for unawareness in non-standard state-space model, and thus construct environments that allow for bounded rationality. 
One of the ideas behind trying to allow for unawareness in state-space model is to be able to transpose this notion to contract-models.

Different approaches coexist based on their works. For instance, while most models allow for unawareness in terms of knowledge an ignorance, Heifetz et al. (2013) allow for different probabilistic beliefs over the realization of outcomes depending on the awareness of the agents; Meier and Schipper (2012a), for their part, develop Bayesian games with unawareness, with the introduction of a Nash equilibrium with unawareness, and define some equilibrium refinements.

Modeling the impact of asymmetric awareness on probabilistic beliefs several agents are aware of allows in particular to design models with moral-hazard under unawareness.

In this line, Zhao (2008) considers a contract with moral-hazard and unawareness where both the principal and the agent can be unaware, while Von Thadden and Zhao (2014) propose a model with a fully aware principal and an unaware agent.

In a more recent paper, Li et al. (2016) study an applied situation involving unawareness, and study when it is interesting for a company to increase the awareness degree of the agents. We refer to Schipper (2014) for a detailed survey of the literature.

The model used here adopts the generalized state space model introduced by Heifetz et al. (2006) and Auster (2013), and extends it with a heterogeneous population of agents. As in Auster (2013), under the assumption of independence of the contingencies, the model respects the justifiability condition required by Filiz-Ozbay (2012), that is, on the agents' point of view, the principal must behave rationally, even if the agents are only partially aware. Our model is in line with the evolution of the research on unawareness, translating from the theoretical structure of unawareness in the agent's expected utility to more applied considerations such as what is the optimal awareness revelation in a contract with asymmetric awareness, with implications for industrial organization. 


\section{The Model}

The model is adapted from Auster (2013), and includes heterogeneity in the consumer's effort cost. The problem is an agency contract, with a profit-maximizing principal, neutral to risk, contracting with utility-maximizing agents who is averse to risk. The agents can be of two types. Agents of type $\theta_{H}$ have a low cost to effort $\mathcal{C}(e)$, while agents of type $\theta_{L}$ : have a high cost to effort $e$.

Assumption 1: $e_{L}>\mathcal{C}\left(e_{L}\right), \Delta e=e_{H}-e_{L}>\Delta \mathcal{C}(e)=\mathcal{C}\left(e_{H}\right)-\mathcal{C}\left(e_{L}\right)$.

This assumption states that for the effort-averse agent, exerting a low effort is more costly than for the other agents. The difference between a high effort and a low effort is bigger for the effort-averse agent. It is coherent with the notion of effort-aversion and allows for more mathematical tractability. Also, let us note $P\left(\theta=\theta_{H}\right)=\alpha$ the proportion of $\theta_{H}$ in the population.

Besides this heterogeneity, the model is strictly identical to Auster (2013), and is described below.

The agents engage in a task for an effort $e$, and with an outcome $y \in \mathcal{Y}=\left\{y_{1}, . ., y_{k}\right\}, y_{i} \in$ $\mathbb{R}$. Both the profit of the principal and the utilities of the agents are derived from the outcome.

The principal derives a profit from the outcome y, and loses profit on the money transfer $C($.$) to the agents. The agents get a positive utility from monetary transfers$ $C($.$) and a negative utility from exerting an effort e$. The outcome is observable and verifiable. While the outcomes are fixed, $C(y)$ is a variable that the principal can use in order to design the contract.

- Principal: $U_{P}(y)=y-C(y)$ where $C: \mathbb{R} \rightarrow \mathbb{R}^{+}$is a compensation function.

- Agents: $U_{A}^{H}(y, e)=V(C(y))-\mathcal{C}(e)$ for the high-type and $U_{A}^{L}(y, e)=V(C(y))-e$ for the low-type, where $V: \mathbb{R} \rightarrow \mathbb{R}$ verifies the Inada conditions $]^{1]} e \in\left\{e_{H}, e_{L}\right\} ; e_{H}>$

\footnotetext{
${ }^{1}$ For $x \in\left[0,+\infty\left[, \mathrm{V}\right.\right.$ is continuously differentiable; $\mathrm{V}(0)=0 ; \mathrm{V}^{\prime}(\mathrm{x})>0 ; \mathrm{V}^{\prime \prime}(\mathrm{x})<0 ; \lim _{x \rightarrow+\infty} V^{\prime}(x)=0$; $\lim _{x \rightarrow 0} V^{\prime}(x)=+\infty$.
} 
$e_{L}$ is a costly effort that the agents exert and that affects the probability of occurrence of $y$.

Let us denote by $\Upsilon=\left\{v_{1}, v_{2}, \ldots, v_{n}\right\}$, with $v_{i}=0$ or 1 a set of contingencies affecting the outcome. Out of this set is derived the set of states of the world $S=\left\{s_{1}, . ., s_{2}|\Upsilon|\right\}$ composed by the combinations of the contingencies. The agents perceive $\Upsilon_{A} \subseteq \Upsilon$ and $S_{A} \subseteq S$ because of their unawareness. As an illustration, let us consider Auster's example on the state of the world structure: for $\Upsilon=\left\{v_{1}, v_{2}\right\}$ and $\Upsilon_{A}=\left\{v_{1}\right\}$, the resulting sets are: $S=\{(0,0),(1,1),(1,0),(0,1)\}$ and the one perceived by the agent is $S_{A}=\{(0),(1)\}$.

Assumption 2: The random variables $v$ and $v^{\prime}$ are conditionally independent given $e$ for any $v, v^{\prime} \in \Upsilon$.

This assumption is crucial in Auster's set-up, for it allows the contract to respect the justifiability constraint. Thanks to it, from the agent's point of view, the principal is always perceived as rational. This is not always the case if contingencies are not independent.

Uncertainty occurs by the way of a mapping $M: S \rightarrow \mathcal{Y}$ that defines for each $s$ a lottery over the set $\mathcal{Y} ; y=f(s)$, with $f$ a density function. Introducing probabilities illustrates the fact that outcomes are stochastic for a given state of the world. The mapping perceived by the agent is defined in the following way: $y=\hat{f}(s)=f(s, 0, . ., 0)$ for $s \in \hat{S}$.

Stochasticity is also introduced at the contingency level. The agents' effort impacts the values of the contingencies $\left(P\left(v_{i}=1 \mid e_{H}\right)\right.$ is not always equal to $P\left(v_{i}=1 \mid e_{L}\right)$ for $\left.v_{i} \in \Upsilon\right)$. To sum-up, the probability of occurrence of an outcome y, $P\left(y \mid e_{H}\right)$ is in general different from $P\left(y \mid e_{L}\right)$. The agents exert an effort that increases the chances of some contingency to take a certain value, and a distribution of probability of occurrence over the set of outcome is inferred from the resulting state of the world.

The notion of effort here is associated with costly actions for the agents that increase the probabilities of occurrence of outcomes profitable for the principal. For some outcomes $Y$, we have $P\left(Y \mid e_{H}\right)>P\left(Y \mid e_{L}\right)$ and overall $E\left[Y \mid e_{H}\right]>E\left[Y \mid e_{L}\right]$. 
In this setting, following Auster (2013), considering our objective function it is assumed that it is always preferable to implement a high effort. However, implementation of a low effort by one type of the agents may occur, in order for the principal to distinguish the types of the agents.

The principal computes expected utilities using the whole set $\Upsilon$ and thus the full sets $S$ and $\mathcal{Y}$. The notion of awareness is introduced here in the way the agents compute their expected utilities. Indeed, they only use part of the sets to infer the probabilities. More precisely, they compute it over the set $\Upsilon_{A} \subseteq \Upsilon$, with $v_{i}=0$ if $v_{i} \in \Upsilon \backslash \Upsilon_{A}$. Note that it is assumed that both agents always have the same degree of awareness. The principal can not offer contracts with different degrees to the agents. This assumption fits with a screening behavior of the agents when screening is costless.

Following Filiz-Ozbay (2012) and Auster (2013), we define a contract in our set-up.

Definition 1: A contract is a triplet $(\hat{\Upsilon}, C, e)$, with $\Upsilon_{A} \subseteq \hat{\Upsilon} \subseteq \Upsilon, C: \mathcal{Y} \rightarrow \mathbb{R}^{+}$and $e \in\left\{e_{L}, e_{H}\right\}$

The notion of incomplete contract given by Filiz-Ozbay (2012) and Auster (2013) is also introduced.

Definition 2: A contract $(\hat{\Upsilon}, C, e)$ is incomplete if $\hat{\Upsilon} \neq \Upsilon$, otherwise it is complete.

The principal and the agents play the following game:

1. The principal proposes a contract over the outcome $y$, with two parameters $(\hat{\Upsilon}, C(y))$ where $\Upsilon_{A} \subseteq \hat{\Upsilon} \subseteq \Upsilon$.

2. The agents learn their types and choose a level of effort $e \in\left\{e_{H}, e_{L}\right\}$.

3. A stochastic outcome $y$ occurs depending on the states of the world.

4. The principal pays $C(y)$, depending on the $y$ that occurred.

The principal designs the contract following his expected profit,

$$
E\left[U_{P}(y, e, C(.))\right]=\sum_{y \in Y} P(y \mid e)[y-C(y)]
$$


Similarly, the agents decide to accept or reject it considering their expected utilities,

$$
\begin{gathered}
E_{\hat{Y}}\left[U_{A}^{L}(y, e, C(.))\right]=\sum_{y \in \hat{Y}} \hat{P}(y \mid e) V(C(y))-e \\
E_{\hat{Y}}\left[U_{A}^{H}(y, e, C(.))\right]=\sum_{y \in \hat{Y}} \hat{P}(y \mid e) V(C(y))-\mathcal{C}(e)
\end{gathered}
$$

They compute it depending on their degree of awareness. We normalize reservation profit and utility to zero, below which the principal or the agents do not accept the contract.

$\hat{P}\left(y \mid e_{H}\right)$ is the perceived probability of occurrence of outcome $y$ for a reduced contingencyset size $\hat{\Upsilon}$ (associated with reduced sets $\hat{S}$ and $\hat{\mathcal{Y}}$ ) that the agent is made aware of by the principal as part of the contract.

The perceived-probability associated with incomplete awareness is computed thanks to Bayes formula: $\hat{P}(y \mid e):=\frac{P(y \mid e)}{P(\hat{v} \mid e)}$ for $y \in \hat{\mathcal{Y}}$ where $P(\hat{v} \mid e)=\prod_{v \notin \hat{v}} P[v=0 \mid e]$.

We now solve the agency problem in the contract with observable efforts, and find the optimal strategy for the principal, and the resulting level of awareness.

\section{Equilibrium contract: observable efforts}

We first solve the problem with observable efforts as a benchmark, which also gives us insights on the impact of monitoring technologies such as IoT in markets with unawareness. We treat the case with hidden types, since observable types allow the principal to perfectly discriminate between the agents, and thus gives the exact same results as in Auster (2013) for this case.

Note that the results still verify the justifiability constraint, by the same arguments as Auster relying on the assumption of independence of the contingencies.

The principal wants to maximize his utility under constraints that differ depending on the strategy that he chooses. Namely, the problem is to choose the one that gives him the higher profit among the three following ones:

1. Pooling equilibrium, with identical compensation for both types. 
2. Excluding equilibrium where only $\theta_{H}$ is served.

3. Separating equilibrium based on different implementations of efforts depending on the type: $\theta_{L}$ implements $e_{L}, \theta_{H}$ implements high effort at $\operatorname{cost} \mathcal{C}\left(e_{H}\right)$. (the opposite would necessarily be sub-optimal, since the effort-cost gap is bigger for $\theta_{L}$ )

\section{Pooling equilibrium contract:}

$$
\begin{aligned}
& \max _{\hat{v}, C(.)}\left\{E\left[U_{P}\left(y, e_{H}, C(.)\right)\right]\right\} \\
& \text { s.t. : } P C_{L}: E_{\hat{Y}}\left[U_{A}^{L}\left(y, e_{H}, C(.)\right)\right] \geq 0
\end{aligned}
$$

The principal proposes one contract that respects the participation constraints of both agents' types. $P C_{L}$ implies that $\theta_{H}$ agents participates.

\section{Excluding equilibrium contract:}

$$
\begin{aligned}
& \max _{\hat{v}, C_{e, H}(.)}\left\{\alpha E\left[U_{P}\left(y, e_{H}\right), C_{e, H}(.)\right]\right\} \\
& \text { s.t. : } P C_{H}: E_{\hat{Y}}\left[U_{A}^{H}\left(y, e_{H}, C_{e, H}(.)\right)\right] \geq 0
\end{aligned}
$$

The principal proposes one contract that satisfies the constraints of $\theta_{H}$ only. $\theta_{L}$ do not participate and only a proportion $\alpha$ of the population is served.

\section{Separating equilibrium contract:}

$$
\begin{aligned}
& \max _{\hat{v}, C_{H}(.), C_{L}(.)}\left\{\alpha E\left[U_{P}\left(y, e_{H}\right), C_{s, H}(.)\right]+(1-\alpha) E\left[U_{P}\left(y, e_{L}\right), C_{s, L}(.)\right]\right\} \\
\text { s.t. }: & P C_{\theta}: E_{\hat{Y}}\left[U_{A}^{\theta}\left(y, e_{\theta}, C_{s, \theta}(.)\right)\right] \geq 0 \\
& I C_{\theta}: E_{\hat{Y}}\left[U_{A}^{\theta}\left(y, e_{\theta}, C_{s, \theta}(.)\right)\right] \geq E_{\hat{Y}}\left[U_{A}^{\theta}\left(y, e_{-\theta}, C_{s,-\theta}(.)\right)\right]
\end{aligned}
$$

Besides ensuring the participation of both agent's types, the principal has to motivate the agents to exert different effort levels that allow him to distinguish them. Intuitively, he has $\theta_{L}$ exerting a low effort, and inversely for $\theta_{H}$. Two other constraints are added to the initial ones that concern the effort level.

Non-negative compensation is required in each problem.

Proposition 1.a: A contract with observable efforts and hidden types is always incomplete with minimum awareness disclosure by the principal: $\hat{\Upsilon}^{o}=\Upsilon_{A}$. This holds whatever the strategy adopted by the principal. 
Proof: See Appendix Proof 1.a.

This result is in line with the one of Auster (2013) where the same effect of tightening of the participation constraint happens when contingencies are disclosed.

Proposition 1.b: There exist $\alpha_{p-s}^{o}, \alpha_{s-e}^{o} \in[0,1]$ such as if $\alpha_{p-s}^{o}<\alpha_{s-e}^{o}$ the principal chooses among the following strategies:

- One unique contract pooling the agents for $\alpha \in\left[0, \alpha_{p-s}^{o}\right]$

- Two separated contracts for $\alpha \in\left[\alpha_{p-s}^{o}, \alpha_{s-e}^{o}\right]$

- One excluding contract for $\alpha \in\left[\alpha_{s-e}^{o}, 1\right]$

Proof: See Appendix Proof 1.b.

If $\alpha_{p-s}^{o} \geq \alpha_{s-e}^{o}$, only pooling and excluding contract are possible.

Pooling implies that the principal loses revenue from $\theta_{H}$, whose utility is not fully exploited. But since the principal does not identify the type of the agent, he does not lose revenue on the identification. The lower the $\alpha$, the less he loses on $\theta_{H}$, since they are less represented in the population. On the opposite, for a high proportion of $\theta_{H}$, it is less profitable for the principal to separate the agents, since it adds a constraint to the contract, while serving only $\theta_{H}$ and excluding $\theta_{L}$ allows for full extraction of $\theta_{H}$, and no separation cost. Excluding can thus be more profitable than separating, despite the fact that profits are made over a proportion $\alpha$ of the population only.

\section{Equilibrium contract: hidden efforts}

We now look at the problem with hidden efforts, where the types are still hidden. In this problem with hidden efforts, the principal still wants to maximize his utility choosing between pooling, excluding and separating the agents.

The problems are the following: 


\section{Pooling-equilibrium contract:}

$$
\begin{gathered}
\max _{\hat{v}, C(.)}\left\{E\left[U_{P}\left(y, e_{H}\right), C(.)\right]\right\} \\
\text { s.t. : } \quad P C_{L}: E_{\hat{Y}}\left[U_{A}^{L}\left(y, e_{H}, C(.)\right)\right] \geq 0 \\
\\
I C_{L}: E_{\hat{Y}}\left[U_{A}^{L}\left(y, e_{H}, C(.)\right)\right] \geq E_{\hat{Y}}\left[U_{A}^{L}\left(y, e_{L}, C(.)\right)\right]
\end{gathered}
$$

To the initial pooling contract is added the need for the principal to motivate a high effort. Again, motivating it for $\theta_{L}$ intuitively implies it for $\theta_{H}$.

\section{Excluding-equilibrium contract:}

$$
\begin{aligned}
& \max _{\hat{v}, C_{e, H}(.)}\left\{E\left[U_{P}\left(y, e_{H}\right), C_{e, H}(.)\right]\right\} \\
\text { s.t. : } & P C_{H}: E_{\hat{Y}}\left[U_{A}^{L}\left(y, e_{H}, C_{e, H}(.)\right)\right] \geq 0 \\
& I C_{H}: E_{\hat{Y}}\left[U_{A}^{L}\left(y, e_{H}, C_{e, H}(.)\right)\right] \geq E_{\hat{Y}}\left[U_{A}^{L}\left(y, e_{L}, C_{e, H}(.)\right)\right]
\end{aligned}
$$

The principal faces the same problem of agent-participation and motivation as above, but only cares about having $\theta_{H}$ respecting it. As in the observable-effort contract, excluding $\theta_{L}$ implies that he only makes profits over $\alpha$ of the population.

\section{Separating-equilibrium contract:}

$$
\begin{aligned}
& \max _{\hat{v}, C_{s, H}(.), C_{s, L}(.)}\left\{\alpha E\left[U_{P}\left(y, e_{H}\right), C_{s, H}(.)\right]+(1-\alpha) E\left[U_{P}\left(y, e_{L}\right), C_{s, L}(.)\right]\right\} \\
\text { s.t. : } & P C_{\theta}: E_{\hat{Y}}\left[U_{A}^{\theta}\left(y, e_{\theta}, C_{s, \theta}(.)\right)\right] \geq 0 \\
& I C_{\theta}^{e}: E_{\hat{Y}}\left[U_{A}^{\theta}\left(y, e_{\theta}, C_{s, \theta}(.)\right)\right] \geq E_{\hat{Y}}\left[U_{A}^{\theta}\left(y, e_{-\theta}, C_{s, \theta}(.)\right)\right] \\
& I C_{\theta}^{\text {type }}: E_{\hat{Y}}\left[U_{A}^{\theta}\left(y, e_{\theta}, C_{s, \theta}(.)\right)\right] \geq E_{\hat{Y}}\left[U_{A}^{\theta}\left(y, e_{-\theta}, C_{s,-\theta}(.)\right)\right]
\end{aligned}
$$

Again the principal has to ensure the participation of both types of agents. He also has to ensure that the agents choose the right contract $\left(I C_{\theta}^{\text {type }}\right)$, and that once this contract is chosen they exert the effort contracted on $\left(I C_{\theta}^{e}\right)$.

Solving for these problems gives us the following propositions.

Proposition 2: In the contract with hidden efforts and types, there exist $\alpha_{p-s}^{h} \geq$ 0 and $\alpha_{s-e}^{h}$, such that if $\alpha_{p-s}^{h}<\alpha_{s-e}^{h} \leq 1$ the principal chooses the following strategies and awareness level: 
- One unique contract pooling the agents for $\alpha \in\left[0, \alpha_{p-s}^{h}\right]$ with awareness $\hat{\Upsilon}_{p}^{h}$.

- Two separated contracts for $\alpha \in\left[\alpha_{p-s}^{h}, \alpha_{s-e}^{h}\right]$, with $\hat{\Upsilon}_{s}^{h}$.

- One excluding contract for $\alpha \in\left[\alpha_{s-e}^{h}, 1\right], \hat{\Upsilon}_{e}^{h}$.

Else, the principal chooses between pooling and excluding.

Also, we have $\Upsilon_{A} \subseteq \hat{\Upsilon}_{e}^{h}, \hat{\Upsilon}_{s}^{h}, \hat{\Upsilon}_{p}^{h}$

Proof: See Appendix Proof 2 .

In general awareness is not comparable between the contracts. However, conditions under which awareness is higher in a contract than in another can be derived. Let us consider the conditions under which awareness extension is profitable for the principal. We consider two contracts, one with no awareness disclosure and one with an extended awareness $\hat{\Upsilon}$. For the pooling and excluding equilibrium, similarly to Auster's explanation of the awareness disclosure's trade-off, the profit differences between the contracts with $\hat{\Upsilon}$ is:

$$
\Delta \Pi_{p, \hat{\Upsilon}-\Upsilon_{A}}=\prod_{v \notin \Upsilon_{A}} P\left[v=0 \mid e_{H}\right] E_{\Upsilon_{A}}\left[C^{\Upsilon_{A}}(y) \mid e_{H}\right]-\prod_{v \notin \hat{\Upsilon}} P\left[v=0 \mid e_{H}\right] E_{\hat{\Upsilon}}\left[C^{\hat{\Upsilon}}(y) \mid e_{H}\right]
$$

for the pooling contract,

$$
\Delta \Pi_{e, \hat{\Upsilon}_{-} \Upsilon_{A}}=\prod_{v \notin \Upsilon_{A}} P\left[v=0 \mid e_{H}\right] E_{\Upsilon_{A}}\left[C_{e}^{\Upsilon_{A}}(y) \mid e_{H}\right]-\prod_{v \notin \hat{\Upsilon}} P\left[v=0 \mid e_{H}\right] E_{\hat{\Upsilon}}\left[C_{e}^{\hat{\Upsilon}}(y) \mid e_{H}\right]
$$

for the excluding contract, and

$$
\begin{array}{r}
\Delta \prod_{s, \hat{\Upsilon}-\Upsilon_{A}}=\alpha\left\{\prod_{v \notin \Upsilon_{A}} P\left[v=0 \mid e_{H}\right] E_{\Upsilon_{A}}\left[C_{s, H}^{\Upsilon_{A}}(y) \mid e_{H}\right]-\prod_{v \notin \hat{\Upsilon}} P\left[v=0 \mid e_{H}\right] E_{\hat{\Upsilon}}\left[C_{s, H}^{\hat{\Upsilon}}(y) \mid e_{H}\right]\right\} \\
+(1-\alpha)\left\{\prod_{v \notin \Upsilon_{A}} P\left[v=0 \mid e_{L}\right] E_{\Upsilon_{A}}\left[C_{s, L}^{\Upsilon_{A}}(y) \mid e_{L}\right]-\prod_{v \notin \hat{\Upsilon}} P\left[v=0 \mid e_{L}\right] E_{\hat{\Upsilon}}\left[C_{s, L}^{\hat{\Upsilon}}(y) \mid e_{L}\right]\right\}
\end{array}
$$

for the separating contract. We note as in Auster $\Delta C_{\Upsilon_{A}}^{\hat{\Upsilon}_{A}}=E_{\Upsilon_{A}}\left[C^{\Upsilon_{A}}(y) \mid e_{H}\right]-$ $E_{\hat{\Upsilon}}\left[C^{\hat{\Upsilon}}(y) \mid e_{H}\right]$, moreover,

$\Delta P_{L, \Upsilon_{A}}^{\hat{\Upsilon}}=\prod_{v \notin \hat{\Upsilon}} P\left[v=0 \mid e_{L}\right]-\prod_{v \notin \Upsilon_{A}} P\left[v=0 \mid e_{L}\right]$

For a given awareness $\hat{\Upsilon}$, awareness is increased for the following necessary and sufficient conditions:

Pooling equilibrium:

$$
\Delta C_{p, \Upsilon_{A}}^{\hat{\Upsilon}} \geq\left(1-\prod_{v \in \hat{\Upsilon} \backslash \Upsilon_{A}} P\left[v=0 \mid e_{H}\right]\right) E_{\Upsilon_{A}}\left[C_{p, h}^{\Upsilon_{A}}(y) \mid e_{H}\right]
$$

$\underline{\text { Separating equilibrium: }}$ 


$$
\Delta C_{s, \Upsilon_{A}}^{\hat{\Upsilon}}-\frac{1-\alpha}{\alpha} V^{-1}\left(e_{L}\right) \frac{\Delta P_{\Upsilon_{A}}^{\hat{\Upsilon}}}{\prod_{v \notin \hat{\Upsilon}} P\left[v=0 \mid e_{H}\right]} \geq\left(1-\prod_{v \in \hat{\Upsilon} \backslash \Upsilon_{A}} P\left[v=0 \mid e_{H}\right]\right) E_{\Upsilon_{A}}\left[C_{s, h}^{\hat{\Upsilon}}(y) \mid e_{H}\right]
$$

Excluding equilibrium:

$$
\Delta C_{e, \Upsilon_{A}}^{\hat{\Upsilon}} \geq\left(1-\prod_{v \in \hat{\Upsilon} \backslash \Upsilon_{A}} P\left[v=0 \mid e_{H}\right]\right) E_{\Upsilon_{A}}\left[C_{e, h}^{\Upsilon_{A}}(y) \mid e_{H}\right]
$$

These conditions are similar to the ones derived by Auster, where $\Delta C_{\Upsilon_{A}}^{\hat{\Upsilon}}$ represent the information gain, and $\left(1-\prod_{v \in \hat{\Upsilon} \backslash \Upsilon_{A}} P\left[v=0 \mid e_{H}\right]\right) E_{\Upsilon_{A}}\left[C_{p, h}^{\Upsilon_{A}}(y) \mid e_{H}\right]$ is the participation loss. It is natural to find these results for the pooling and excluding equilibrium where the structure of the problem is not changed. For the separating equilibrium, the constant compensation for the low types decreases the information gain since part of it must be compensated. The impact of this limitation is weighted by the proportion of low types $\frac{1-\alpha}{\alpha}$. The trade-off remains, and thus Auster's result holds for the separating contract.

The figures below illustrates cases where awareness is optimally higher in each contract compared to the others:.$^{2}$

Apart from these results, we give a sufficient condition illustrating the possibility for the separating contracts to occur more often in the observable-effort case.

Assumption 3 : We assume that the awareness structure and optimal compensation scheme follow the rule in Appendix.

Proposition 3: Under Assumption 3, the different $\alpha$ at the frontier between two strategies in equilibrium change between the observable and hidden effort contracts, and follow the ranking below:

$$
0 \leq \alpha_{p-s}^{o} \leq \alpha_{p-s}^{h} \leq \alpha_{s-e}^{h} \leq \alpha_{s-e}^{o} \leq 1
$$

\section{Proof: See Appendix Proof 3 .}

This specific case illustrates the change of strategy that may occur when a monitoring technology enters the market. Here firms that acquire such a technology sometimes skip from proposing a pooling contract to proposing a separating one. For other agents' proportions, the change may be from an excluding contract to a pooling one. The implication of this change depends on the nature of the markets and are discussed below.

\footnotetext{
${ }^{2}$ Again we transpose Auster's example with
} 


\section{Extension: duopoly market}

Let us now study the impacts of competition on the market structures and equilibrium. It is assumed that both firms know the same set of contingencies, and several cases of competition are considered: a duopoly with both firms and hidden efforts; a duopoly where one of the firms has a technology allowing to observe the effort while the other does not; and a duopoly where both firms can monitor, and efforts are thus observable. The degree of awareness-disclosure is identical in both contracts proposed by the firms. This constraint results from the rational screening behavior of an economic agent facing two different contracts, and is then more realistic. From now on, let us note Firm A the firm that observes the efforts of the agents, and firm B the one that does not.

\subsection{Case 1 and 2: symmetric competition}

Both situations of symmetric competition are studied here: with and without the monitoring technology. Naturally, symmetric competition leads to zero profits for both firms, following a Bertrand mechanism. This zero-profit result is analyzed by Auster (2013). The questions that remain concern the type of contract proposed to the agents, and the resulting awareness disclosure.

Proposition 4.a: If firms contract with observable efforts:

1. Awareness is necessarily maximal

2. Profits are entirely redistributed

3. The contract offered is pooling

Proof: Bertrand competition is equivalent for the firms to maximize the agents' utilities under limited-liability constraint. This problem has full redistribution of the profits and full awareness for solution, in an observable effort set-up. See Appendix Proof 4.a for a proof of this result. Consider an equilibrium where the firms offer partial awarenessdisclosure to the agents, then, a firm that deviates to full awareness and almost-full 
redistribution necessarily increases the utility of the agents, and gets the whole demand. Thus, the only Nash equilibrium offers full awareness and full profits redistribution.

Suppose that the contract is excluding. Then, a symmetric equilibrium implies that both firms make zero profits over the part of the population that is served, and do not serve the other part. It is thus optimal for the firms to deviate from the equilibrium and serve the excluded part of the population. Since it is assumed that exerting a high effort is in general more profitable for the firm and for the agents, both firms offer a contract that fits with the high-type-agent. Thus, every agent is offered the same pooling contract.

Assume now that the contract is separating, again both firms make zero profits on both parts of the population. It is then optimal for them to deviate to a pooling contract where they lower their constraint compared to both initial contracts. Pooling the contracts is again the equilibrium solution.

Proposition 4.b: If firms contract with hidden efforts, there exist a partial awareness degree below which no contract is a Nash equilibrium, and above which to every degree of awareness, full awareness included, corresponds a compensation scheme that makes the contract a Nash equilibrium.

Proof: Again, this result comes from computing the contract that maximizes the agents' utilities under limited-liability constraint of the company and incentive-compatibility constraint. In monopoly, this problem leads to partial awareness in a world with hidden effort, with an optimal degree of awareness corresponding to the threshold one mentioned above. This result is proved in Appendix Proof 4.b.

Consider an equilibrium where both firms propose a degree of awareness below the optimal one. They both make zero profits. The firms deviate then to the optimal degree of awareness that maximizes the agents' utilities for complete redistribution. Both firms deviate, since if one of the firms remains with the initial contract the other one can lower the retribution and still capture the whole demand. Any contract with an awareness disclosure below the optimal one is not an equilibrium.

Consider now an equilibrium where both firms have the same awareness disclosure, 
above the optimal one. A firm that deviates to a lower awareness contract decreases the agents' utilities, since the agents now compute their utilities with their extended awareness level. On the other hand, deviating to a higher level of awareness is not necessarily optimal for the firms, as stated in Auster (2013). We can note that full awareness is also an equilibrium, from the arguments developed above.

By the same mechanism as in the observable effort contract, the equilibrium contract pools the agents.

\subsection{Case 3: asymmetric competition}

Let us look at the awareness-symmetric equilibrium in a competition asymmetric over the technology. One firm contracts with observable efforts while it is hidden for the other firm. We start by tackling the question of the nature of the contract, then we focus on the awareness disclosure.

Proposition 5.a: Firm B makes zero profit, Firm A makes profit identically to a monopolist constrained on awareness and profits.

\section{Proof:}

As in the technology-symmetric competition, assume that the firms are in an excluding equilibrium. Then the problem that they face, in order to maximize the agents' utilities and get the whole demand is for Firm A:

$$
\begin{aligned}
& \max _{\hat{v}, C(.)}\left\{E\left[U_{P}\left(y, e_{H}\right), C(.)\right]\right\} \\
& \text { s.t. : } P C: E_{\hat{Y}}\left[U_{A}^{L}\left(y, e_{H}, C(.)\right)\right]=0
\end{aligned}
$$

While Firm B wants to solve the same problem with hidden efforts:

$$
\begin{aligned}
& \max _{\hat{v}, C(.)}\left\{E\left[U_{P}\left(y, e_{H}\right), C(.)\right]\right\} \\
& \text { s.t. : } P C: E_{\hat{Y}}\left[U_{A}^{L}\left(y, e_{H}, C(.)\right)\right]=0 \\
& I C: E_{\hat{Y}}\left[U_{A}^{L}\left(y, e_{L}, C(.)\right)\right]=0
\end{aligned}
$$


A Bertrand competition with asymmetric information on efforts drives the profits down until Firm B cannot increase the agents' utilities without violating the IC constraint. This maximal awareness corresponds to the threshold awareness in the symmetric duopoly. At this stage, Firm A can get the whole demand by increasing the compensation by a small value $\epsilon$. Note that again, a higher level of awareness corresponds to a Nash equilibrium, with zero profits for Firm B.

Eventually, Firm A faces the problem of maximizing its profits, under a reservation utility for the agents corresponding to the utility they get by turning to Firm B, tightening the PC constraint.

Proposition 5.b: The contracts proposed by the firms are necessary pooling.

\section{Proof:}

Assume that both firms exclude the low-type agent. Firm B by making zero profits in the equilibrium as described above, serves the low-type agent. Again, following our assumption that the high effort is optimal for the principal and the agents, Firm B proposes a high effort contract, that is a better alternative for the high-type agent than the one offered by Firm A (it is immediate to show by rewriting the optimization problems).

Again, if both firms propose separating contracts, Firm B makes zero profits, and thus has interest to deviate to pooling contracts, for it lowers the constraints compared to the initial contracts. It intensifies the competitive pressure on Firm A that has to propose also a pooling contract.

Eventually, one pooling contract is offered to the agents.

It has been shown that competing implies no profits for the firms, however, situations occur where the firms can anticipate this zero-profit result, and cover the market in such way that they make positive profits. I study such a situation in the section below. 


\subsection{Case 4: Optimal market structure with a historical actor and a new entrant.}

In this sub-section, a two-stage game is considered, where one of the firms is in monopoly in the first step and the other firm enters the market at the second step.

We want to show that this sequential structure allows the firms to make positive profits. This game is solved in this regard. As shown above it is not profitable for the new entrant to start a frontal competition with the historical player. Specifically, if firms are technology-symmetric, direct competition leads both of them to zero profits. It is thus optimal for the historical player (present in stage 1) to leave some room to the new entrant.

By backward induction, the traditional player designs its contracts anticipating that a new entrant arrives at stage 2 .

Let us see what strategy is optimal in stage 2 :

- If the contract offered in stage 1 is pooling, separating, or excluding under the constraints used until now, Bertrand competition occurs, leading to zero profits.

- If the contract offered in stage 1 is excluding, with the additional separating constraint that the high-type agents do not skip to a hypothetical second contract for the low-type, taking the form of a separating contract, it is optimal for the second player to propose a low-type agents low-effort contract, which results in a separation of the market in the same way as in the previous separating contracts, each firm dealing with one type of agents.

- If the contract offered in stage one is pooling, with exertion of a low effort, in the same fashion as in the previous separating contracts, the second agent can offer a high-effort contract with the constraint that only high-type agents accept it, and the market has the same separating structure as above, but with the second player dealing with the high-type agents. 
The historical player chooses either strategy to maximize his profits in the first stage, and to make zero profits in the second, or to lower its profits in the first stage and make positive ones in the second, depending on its discount factor. Also, the firm either proposes an excluding contract, or a low-effort pooling one depending on the proportion of the agents. If there is room in the market, the new entrant avoids frontal competition whatever the proportion of the agents, and makes positive profits. It implies that constraints appear for both firms, that correspond to the ones in separating contracts.

If the traditional player owns the technology, he might accept frontal competition, to the extent that the competitive pressure is low enough. Similarly, if the new entrant is the one with the technology, he might start frontal competition also, under conditions of the same nature.

\section{Discussion}

Allowing for awareness asymmetry in moral-hazard models with heterogeneous agents allows for a better understanding of market structures and a better evaluation of the consumer's utilities and the firm profits. The two factors of high interest in our set-up, that are the strategy of the firms and the degree of awareness given in the contracts, are altered compared to the traditional model.

Comparing our set-up with the traditional one without unawareness shows several consequences. We first notice that the contracts without unawareness are included in our set-up and corresponds to the case where $\Upsilon=\Upsilon_{A}$.

A first effect that's quite immediate to observe is that, since disclosing awareness is a non-financial lever for the firm to increase its profits, the utilities of the agents in contracts with unawareness are always below the utilities with symmetric awareness. We can thus start by noting that the agent's utilities decrease compared to the traditional set-up.

Taking into account unawareness changes the market structures for some proportions of low types in the population, and shows that under assumption 3, pooling and ex- 
cluding equilibrium occur in more situations than the symmetric awareness set-ups were proving. Again this result is crucial for regulators, especially in markets such as the health-insurance market.

Turning to the comparison between observable and hidden efforts set-ups in contract with unawareness, it is shown that introducing a monitoring technology strongly affects the degree of awareness disclosure, the awareness being automatically driven down to its minimum possible. In the hidden effort environment, this awareness takes different values that are always above the minimum. To that extent the introduction of the technology is a major drawback for the consumers.

Considering now the market structure, under assumption 3 the technology widens the case where separating contracts are chosen by the principal. This occurs in two ways, by proposing pooling contracts in less cases, and similarly for excluding contracts.

The analysis of several configurations of competition offers various results. Symmetric firms moving simultaneously end-up proposing a pooling contract with full awareness in the observable effort case, and partial awareness disclosure in the hidden effort case. Full disclosure is a Nash equilibrium, and, in any situation, firms make zero-profit. When firms are technology asymmetric, or when one firm moves before the other, equilibrium exist where the technology owner or the first mover makes positive profits.

The interpretation of these results clearly depends on the nature of the market studied, the implications of more separating contracts being incomparable between the loan market and the market for health-insurance for instance. In the health-insurance market, the dominant paradigm is risk-pooling at the society level. Offering contracts that allow to discriminate between two types of agents violates this paradigm, to a further extent if one of the agents is encouraged to exert a low effort in order to maximize the firm's profits. This question is central since, with the introduction of the IoT in the health insurance market, and the use of data to design specific contracts in the US, more separating contracts are expected. 


\section{Conclusion}

By introducing unawareness in contract-models with heterogeneous agents, we derive some strong implications on economic agents' utilities and market mechanisms, with a significant social impact. Namely, it is shown that, whatever the strategy, the initial results of Auster hold. While its is direct to see it for the pooling and excluding equilibrium, we prove that the same trade-off exists for separating contracts, and partial awareness disclosure may also occur.

Comparing our results with the one of contract-model with unawareness and homogeneous agents allows us to capture the implications of unawareness on the structures of the market. In particular, while with homogeneous agents the market was always covered, it is shown here that several cases may occur where excluding part of the agents from contracting is optimal for the principal.

Eventually, the equilibrium in competition for several configurations of the use of a monitoring technology are clarified. Also, it is shown that, in sequential games, collusion can occur, leading to a higher extraction of the agents' surplus.

This paper is in line with the trend of the literature, that looks for more applicability of the results of the theory of unawareness and incomplete contracts to industrial organization. More specifically, we believe that considering markets such as health-insurance with the scope of awareness-asymmetric contracts offers a more precise understanding of the process of contract-design. Comparing the market equilibrium in the observable and hidden effort case world helps to understand the changes that occur with the introduction of monitoring technologies.

\section{References}

[1] Sarah Auster. Asymmetric awareness and moral hazard. Games and Economic Behavior, 82:503-521, 2013. 
[2] Eddie Dekel, Barton L Lipman, and Aldo Rustichini. Standard state-space models preclude unawareness. Econometrica, 66(1):159-173, 1998.

[3] Emel Filiz-Ozbay. Incorporating unawareness into contract theory. Games and Economic Behavior, 76(1):181-194, 2012.

[4] Spyros Galanis. Syntactic foundations for unawareness of theorems. Theory and Decision, 71(4):593-614, 2011.

[5] Sanford J Grossman and Oliver D Hart. An analysis of the principal-agent problem. Econometrica: Journal of the Econometric Society, pages 7-45, 1983.

[6] Aviad Heifetz, Martin Meier, and Burkhard C Schipper. Interactive unawareness. Journal of economic theory, 130(1):78-94, 2006.

[7] Aviad Heifetz, Martin Meier, and Burkhard C Schipper. Unawareness, beliefs, and speculative trade. Games and Economic Behavior, 77(1):100-121, 2013.

[8] Bengt Hölmstrom. Moral hazard and observability. The Bell journal of economics, pages 74-91, 1979.

[9] Sanxi Li, Martin Peitz, and Xiaojian Zhao. Information disclosure and consumer awareness. Journal of Economic Behavior \& Organization, 2016.

[10] Eric Maskin and Jean Tirole. Unforeseen contingencies and incomplete contracts. The Review of Economic Studies, 66(1):83-114, 1999.

[11] M Meier and BC Schipper. Conditional dominance in games with unawareness. University of California, Davis, 2012.

[12] Salvatore Modica and Aldo Rustichini. Awareness and partitional information structures. Theory and Decision, 37(1):107-124, 1994.

[13] Burkhard C Schipper. Awareness. Available at SSRN 2401352, 2014.

[14] Ernst-Ludwig von Thadden and Xiaojian Zhao. Multi-task agency with unawareness. Theory and decision, 77(2):197-222, 2014. 
[15] Xiaojian Zhao. Moral hazard with unawareness. Rationality and Society, 20(4):471-496, 2008 .

\section{Appendix}

\section{Proof 1.a:}

Pooling equilibrium:

The proof is the same as in Auster (2013). The profit is

$\Pi\left(\hat{v}, C_{p}^{o}().\right)=\sum_{y \in Y} P\left(y \mid e_{H}\right) y-\sum_{y \in \hat{Y}} P\left(y \mid e_{H}\right) C_{p}^{o}(y)$

Excluding equilibrium:

The proof is again similar, and the profit is:

$\Pi\left(\hat{v}, C_{e}^{o}().\right)=\alpha\left[\sum_{y \in Y} P\left(y \mid e_{H}\right) y-\sum_{y \in \hat{Y}} P\left(y \mid e_{H}\right) C_{e}^{o}(y)\right]$

Separating equilibrium:

We see immediately that $P C_{H}$ is not binding.

$$
\begin{aligned}
& \sum_{y \in \hat{Y}} \hat{P}\left(y \mid e_{H}\right) V\left(C_{H}(y)\right)-\mathcal{C}\left(e_{H}\right) \geq \sum_{y \in \hat{Y}} \hat{P}\left(y \mid e_{L}\right) V\left(C_{L}(y)\right)-\mathcal{C}\left(e_{L}\right) \geq \sum_{y \in \hat{Y}} \hat{P}\left(y \mid e_{L}\right) V\left(C_{L}(y)\right)-e_{L} \geq 0 \\
& \Longrightarrow \sum_{y \in \hat{Y}} \hat{P}\left(y \mid e_{H}\right) V\left(C_{H}(y)\right)-\mathcal{C}\left(e_{H}\right) \geq 0
\end{aligned}
$$

Thus $P C_{L}+I C_{H} \Longrightarrow P C_{H}$.

$$
\begin{aligned}
\max _{\hat{v}, C_{H}(.), C_{L}(.)}\left\{\alpha \sum_{y \in Y} P\left(y \mid e_{H}\right)\left[y-C_{H}(y)\right]+(1-\alpha) \sum_{y \in Y} P\left(y \mid e_{L}\right)\left[y-C_{L}(y)\right]\right\} \\
\text { s.t. : } P C_{L}: \sum_{y \in \hat{Y}} \hat{P}\left(y \mid e_{L}\right) V\left(C_{L}(y)\right)-e_{L} \geq 0 \\
I C_{H}: \sum_{y \in \hat{Y}} \hat{P}\left(y \mid e_{H}\right) V\left(C_{H}(y)\right)-\mathcal{C}\left(e_{H}\right) \geq \sum_{y \in \hat{Y}} \hat{P}\left(y \mid e_{L}\right) V\left(C_{L}(y)\right)-\mathcal{C}\left(e_{L}\right) \\
I C_{L}: \sum_{y \in \hat{Y}} \hat{P}\left(y \mid e_{L}\right) V\left(C_{L}(y)\right)-e_{L} \geq \sum_{y \in \hat{Y}} \hat{P}\left(y \mid e_{H}\right) V\left(C_{H}(y)\right)-e_{H}
\end{aligned}
$$

With respectively $\lambda, \mu, \gamma$ the Lagrange coefficients for $P C_{L}, I C_{H}$ and $I C_{L}$. We show that $\lambda>0, \mu>0, \gamma=0$.

By contradiction: assume $\lambda=0$, then we must have (to respect the Inada condition over $V$ ) simultaneously the strict positivity of $\mu-\gamma$ and $\gamma-\mu$, which is impossible. Thus, $\lambda>0$. 
Then, assume $\mu=0$, then the positivity of the right hand term of the first equation is not respected anymore. Thus, $\mu>0$.

We have: $\sum_{y \in \hat{Y}} \hat{P}\left(y \mid e_{L}\right) V\left(C_{L}(y)\right)=e_{L}$ and $\sum_{y \in \hat{Y}} \hat{P}\left(y \mid e_{H}\right) V\left(C_{H}(y)\right)=\Delta \mathcal{C}(e)+e_{L}$.

Replacing the constant compensations into the binding constraints gives us

$C_{S B}^{H}=V^{-1}\left(e_{L}+\Delta \mathcal{C}(e)\right)$ and $C_{S B}^{L}=V^{-1}\left(e_{L}\right)$.

This optimal allocation is the same with $I C_{L}$ or not, thus $\gamma=0$

Optimal awareness size:

$V^{\prime}\left(C_{H}(y)\right)=\frac{\alpha}{\mu-\gamma} \Pi\left(\Upsilon=0 \mid e_{H}\right)$ and

$V^{\prime}\left(C_{L}(y)\right)=\frac{(1-\alpha)}{\lambda-\mu+\gamma} \Pi\left(\Upsilon=0 \mid e_{L}\right)$

require both to have a minimum awareness size so that $C_{H}$ and $C_{L}$ are as low as possible $\left(\Longleftrightarrow V^{\prime}\left(C_{H}\right)\right.$ and $V^{\prime}\left(C_{L}\right)$ are the bigger possible). Note that compensation over the contingencies the agents are unaware of is necessarily zero.

The profit is:

$\Pi\left(\hat{v}, C_{H, s}^{o}(),. C_{L, s}^{o}().\right)=\alpha \sum_{y \in Y} P\left(y \mid e_{H}\right) y+(1-\alpha) \sum_{y \in Y} P\left(y \mid e_{L}\right) y-\left\{\alpha \sum_{y \in \hat{Y}} P\left(y \mid e_{H}\right) C_{H, s}^{o}(y)+(1-\alpha) \sum_{y \in \hat{Y}} P\left(y \mid e_{L}\right) C_{L, s}^{o}(y)\right\}$

\section{Proof 1.b:}

Which strategy is preferable between separating and pooling?

$$
\begin{aligned}
\Pi\left(\hat{v}, C_{H}(.)\right)-\Pi\left(\hat{v}, C_{H, s}^{o}(.), C_{L, s}^{o}(.)\right) & =(1-\alpha) \sum_{y \in Y} y\left(P\left(y \mid e_{H}\right)-P\left(y \mid e_{L}\right)\right) \\
& +\alpha \sum_{y \in \hat{Y}} P\left(y \mid e_{H}\right)\left(C_{H, s}^{o}(y)-C_{p}^{o}(y)\right) \\
& +(1-\alpha)\left\{\sum_{y \in \hat{Y}} P\left(y \mid e_{L}\right) C_{L, s}^{o}(y)-\sum_{y \in \hat{Y}}\left(P\left(y \mid e_{H}\right) C_{p}^{o}(y)\right)\right\}
\end{aligned}
$$

For $\alpha \geq \alpha_{p-s}^{o}$ separating is preferable, with

$$
\alpha_{p-s}^{o}=\frac{\sum_{y \in Y} y\left(P\left(y \mid e_{H}\right)-P\left(y \mid e_{L}\right)\right)+\sum_{y \in \hat{Y}} P\left(y \mid e_{L}\right) C_{L, s}^{o}(y)-\sum_{y \in \hat{Y}} P\left(y \mid e_{H}\right) C_{p}^{o}(y)}{\sum_{y \in Y} y\left(P\left(y \mid e_{H}\right)-P\left(y \mid e_{L}\right)\right)+\sum_{y \in \hat{Y}} P\left(y \mid e_{L}\right) C_{L, s}^{o}(y)-\sum_{y \in \hat{Y}} P\left(y \mid e_{H}\right) C_{H, s}^{o}(y)}
$$

We show that $\alpha_{p-s}^{o} \in[0,1]$. The positivity of $\alpha_{p-s}^{o}$ is guaranteed by the assumption that high efforts are always more profitable than low efforts for the principal. Let us now consider $1-\alpha_{p-s}^{o}: 1-\alpha_{p-s}^{o} \geq 0 \Longleftrightarrow \sum_{y \in \hat{Y}} P\left(y \mid e_{H}\right) C_{p}^{o}(y) \geq \sum_{y \in \hat{Y}} P\left(y \mid e_{H}\right) C_{H, s}^{o}(y)$. 
Since $\sum_{y \in \hat{Y}} P\left(y \mid e_{H}\right) C_{p}^{o}(y)=V^{-1}\left(e_{H}\right) \geq V^{-1}\left(e_{L}+\Delta \mathcal{C}(e)\right)=\sum_{y \in \hat{Y}} P\left(y \mid e_{H}\right) C_{H, s}^{o}(y)$, $1 \geq \alpha_{p-s}^{o}$ and $\alpha_{p-s}^{o} \in[0,1]$.

Which strategy is preferable between excluding and separating?

$$
\begin{aligned}
& \Pi\left(\hat{v}, C_{e}^{o}(.)\right)-\Pi\left(\hat{v}, C_{H, s}^{o}(.), C_{L, s}^{o}(.)\right)=(1-\alpha)\left[\sum_{y \in \hat{Y}} P\left(y \mid e_{L}\right) C_{L, s}^{o}(y)-\sum_{y \in Y} P\left(y \mid e_{L}\right) y\right] \\
& +\alpha\left[\sum_{y \in \hat{Y}} P\left(y \mid e_{H}\right) C_{H, s}^{o}(y)-\sum_{y \in \hat{Y}} P\left(y \mid e_{H}\right) C_{e}^{o}(y)\right]
\end{aligned}
$$

For $\alpha \geq \alpha_{s-e}^{o}$ excluding is preferable, with

$\forall \alpha \geq \alpha_{s-e}^{o}=\frac{\sum_{y \in Y} P\left(y \mid e_{L}\right) y-\sum_{y \in \hat{Y}} P\left(y \mid e_{L}\right) C_{L, s}^{o}}{\sum_{y \in Y} P\left(y \mid e_{L}\right) y-\sum_{y \in \hat{Y}} P\left(y \mid e_{L}\right) C_{L, s}^{o}+\sum_{y \in \hat{Y}} P\left(y \mid e_{H}\right) C_{H, s}^{o}-\sum_{y \in \hat{Y}} P\left(y \mid e_{H}\right) C_{e}^{o}}$

We show that $\alpha_{s-e}^{o} \in[0,1]$. The positivity of the numerator in guaranteed by the reservation utility of zero for the principal. The same positive term is present at the denominator too, plus $\sum_{y \in \hat{Y}} P\left(y \mid e_{H}\right) C_{H, s}^{o}-\sum_{y \in \hat{Y}} P\left(y \mid e_{H}\right) C_{e}^{o}=V^{-1}\left(e_{L}+\Delta \mathcal{C}(e)\right)-V^{-1}\left(\mathcal{C}(e)_{H}\right) \geq 0$. Let us now consider $1-\alpha_{s-e}^{o}: 1-\alpha_{s-e}^{o} \geq 0 \Longleftrightarrow \sum_{y \in \hat{Y}} P\left(y \mid e_{H}\right) C_{H, s}^{o}-\sum_{y \in \hat{Y}} P\left(y \mid e_{H}\right) C_{e}^{o} \geq$ 0 which is verified above.

Thus $\alpha_{s-e}^{o} \in[0,1]$.

Strategy of the principal

Finally, if $0 \leq \alpha_{p-s}^{o} \leq \alpha_{s-e}^{o} \leq 1$

Consider $\alpha=0$, there is no $\theta_{H}$ agents. Excluding equilibrium leads to zero profits, separating equilibrium leads to profits with the $\theta_{L}$ agents exerting low effort, which is by assumption less profitable than having them exerting a high effort, which corresponds to the pooling equilibrium.

Consider then $\alpha=1$, there is no $\theta_{L}$ agents. Thus pooling equilibrium leads to low profits since high $I C$ and $P C$ constraints apply. Separating equilibrium lowers these constraints, which are still higher than in the excluding contract.

Since the profits are a linear combination of fix sub-profits on each agents type, the total profit evolves continuously with $\alpha$. The principal has to choose between the three strategies.

Else, separating is never more profitable than both excluding and pooling, and the principal does not choose this option. 


\section{Proof 2:}

Let us start by considering the separating equilibrium problem. We then compare the constraints to the ones of the other two configurations, and infer the different levels of awareness disclosure from the constraints.

Separating equilibrium:

It's again immediate to show that $P C_{H}$ is not binding.

FOCs on $C_{H}$ and $C_{L}$ give us, with $\lambda, \mu, \gamma, \theta$ and $\delta$ respectively the Lagrange coefficients of $P C_{L}, I C_{H}^{e}, I C_{L}^{e}, I C_{H}^{\theta}$ and $I C_{L}^{\theta}$.

$$
\begin{aligned}
& C_{H, s}^{H}: \frac{\alpha}{V_{H}^{\prime}}=\frac{1}{P\left(v=0 \mid e_{H}\right)}\left[(\mu+\theta-\delta)-\mu \frac{P\left(v \mid e_{L}\right)}{P\left(v \hat{\mid} e_{H}\right)}\right] \\
& C_{L, s}^{H}: \frac{1-\alpha}{V_{L}^{\prime}}=\frac{1}{P\left(v=0 \mid e_{L}\right)}\left[(\lambda+\delta-\theta+\gamma)-\gamma \frac{P\left(v \mid e_{H}\right)}{P\left(v \mid e_{L}\right)}\right] \\
& \underline{\lambda>0:}
\end{aligned}
$$

Indeed, FOC on $C_{H, s}^{H}$ implies that $\theta-\delta>0$, but then, we necessarily have $\lambda>0$ in the second FOC. Thus, $P C_{L}$ is always binding, and $\sum_{y \in \hat{Y}} \hat{P}\left(y \mid e_{L}\right) V\left(C_{L, s}^{H}(y)\right)=e_{L}$

Note that an immediate condition for this problem to have a solution is to have $e_{H} \geq \Delta \mathcal{C}(e)+e_{L}$

$$
\underline{\theta>0:}
$$

Assume the opposite, then, in the FOC on $C_{H, s}^{H}$, the positivity of the left hand side is not guaranteed.

Thus, $I C_{H}^{\theta}$ is binding, and $\sum_{y \in \hat{Y}} \hat{P}\left(y \mid e_{H}\right) V\left(C_{H, s}^{H}(y)\right)=\Delta \mathcal{C}(e)+e_{L}$. It implies immediately that $I C_{L}^{\theta}$ is slack, and we can remove it from the list of the constraints.

$$
\underline{\mu>0:}
$$

Assume it is not the case, then: FOC on $C_{H, s}^{H}: \frac{\alpha}{V_{H}^{\prime}}=\frac{1}{P\left(v=0 \mid e_{H}\right)}(\theta-\delta)$. This is a constant over y, thus $C_{H, s}^{H}($.$) does not depend on y. But then, V\left(C_{H, s}^{H}\right)=\Delta \mathcal{C}(e)+e_{L}>e_{L}$, and $I C_{H}^{e}$ is violated. Thus $I C_{H}^{e}$ is binding and $\mu>0$.

Let us show now that $I C_{L}^{e}$ is slack. 
Solving the problem

$$
\begin{aligned}
\max _{\hat{v}, C_{H, s}^{H}(.), C_{L, s}^{H}(.)}\left\{\alpha \sum_{y \in Y} P\left(y \mid e_{H}\right)\left[y-C_{H, s}^{H}(y)\right]+(1-\alpha) \sum_{y \in Y} P\left(y \mid e_{L}\right)\left[y-C_{L, s}^{H}(y)\right]\right\} \\
P C_{L}: \sum_{y \in \hat{Y}} \hat{P}\left(y \mid e_{L}\right) V\left(C_{L, s}^{H}(y)\right)=e_{L} \\
I C_{H}^{e}: \sum_{y \in \hat{Y}} \hat{P}\left(y \mid e_{L}\right) V\left(C_{H, s}^{H}(y)\right)=e_{L} \\
I C_{H}^{\theta}: \sum_{y \in \hat{Y}} \hat{P}\left(y \mid e_{H}\right) V\left(C_{H, s}^{H}(y)\right)=\Delta \mathcal{C}(e)+e_{L}
\end{aligned}
$$

Gives us $C_{L, s}^{H}(y)=c s t$

Adding $I C_{L}^{e}: e_{H} \geq \sum_{y \in \hat{Y}} \hat{P}\left(y \mid e_{H}\right) V\left(C_{L, s}^{H}(y)\right)$, the same constant compensation still satisfies the constraints.

Thus this constraint is slack and we are back to problems for which Auster's result apply.

Let us compare the three problems.

$\underline{\text { Pooling }}$

$$
\begin{aligned}
& \max _{\hat{v}, C_{p}^{H}(.)}\left\{\sum_{y \in Y} P\left(y \mid e_{H}\right)\left[y-C_{p}^{H}(y)\right]\right\} \\
& \text { s.t. : } P C: \sum_{y \in \hat{Y}} \hat{P}\left(y \mid e_{H}\right) V\left(C_{p}^{H}(y)\right)=e_{H} \\
& I C: \sum_{y \in \hat{Y}} \hat{P}\left(y \mid e_{L}\right) V\left(C_{p}^{H}(y)\right)=e_{L}
\end{aligned}
$$

\section{$\underline{\text { Separating }}$}

$$
\begin{aligned}
\max _{\hat{v}, C_{H, s}^{H}(.), C_{L, s}^{H}(.)}\left\{\alpha \sum_{y \in Y} P\left(y \mid e_{H}\right)\left[y-C_{H, s}^{H}(y)\right]+(1-\alpha) \sum_{y \in Y} P\left(y \mid e_{L}\right)\left[y-C_{L, s}^{H}(y)\right]\right\} \\
P C_{L}: \sum_{y \in \hat{Y}} \hat{P}\left(y \mid e_{L}\right) V\left(C_{L, s}^{H}(y)\right)=e_{L} \\
I C_{H}^{e}: \sum_{y \in \hat{Y}} \hat{P}\left(y \mid e_{L}\right) V\left(C_{H, s}^{H}(y)\right)=e_{L} \\
I C_{H}^{\theta}: \sum_{y \in \hat{Y}} \hat{P}\left(y \mid e_{H}\right) V\left(C_{H, s}^{H}(y)\right)=\Delta \mathcal{C}(e)+e_{L}
\end{aligned}
$$




\section{Excluding}

$$
\begin{aligned}
& \max _{\hat{v}, C_{e}^{H}(.)}\left\{\alpha \sum_{y \in Y} P\left(y \mid e_{H}\right)\left[y-C_{e}^{H}(y)\right]\right\} \\
& \text { s.t. : } P C: \sum_{y \in \hat{Y}} \hat{P}\left(y \mid e_{H}\right) V\left(C_{e}^{H}\right)(y)=\mathcal{C}_{H} \\
& I C: \sum_{y \in \hat{Y}} \hat{P}\left(y \mid e_{L}\right) V\left(C_{e}^{H}(y)\right)=\mathcal{C}_{L}
\end{aligned}
$$

It is again immediate to prove that the compensation over the contingencies that the agents are not aware of are $C=0$ whatever the type of the agents.

Let us compare the difference in profits for the pooling and separating equilibrium strategy:

Pooling profits:

$$
\Pi_{p}\left(\hat{v}_{p}, C_{p}^{H}(.)\right)=\sum_{y \in Y} P\left(y \mid e_{H}\right) y-\sum_{y \in \hat{Y}_{P}} P\left(y \mid e_{H}\right) C_{p}^{H}(y)
$$

Separating profits:

$$
\begin{aligned}
\Pi_{S}\left(\hat{v}_{S}, C_{H, s}^{H}(.), C_{L, s}^{H}(.)\right)= & \alpha\left\{\sum_{y \in Y} P\left(y \mid e_{H}\right) y-\sum_{y \in \hat{Y}_{S}} P\left(y \mid e_{H}\right) C_{H, s}^{H}(y)\right\} \\
& +(1-\alpha)\left\{\sum_{y \in Y} P\left(y \mid e_{L}\right) y-\sum_{y \in \hat{Y}_{S}} P\left(y \mid e_{L}\right) C_{L, s}^{H}(y)\right\}
\end{aligned}
$$

$\underline{\text { Difference in profits pooling-separating: }}$

$$
\begin{aligned}
\Pi_{P}\left(\hat{v}_{P}, C_{p}^{H}(.)\right)- & \Pi_{S}\left(\hat{v}_{S}, C_{H, s}^{H}(.), C_{L, s}^{H}(.)\right)= \\
& (1-\alpha)\left\{\sum_{y \in Y} P\left(y \mid e_{H}\right) y-\sum_{y \in Y} P\left(y \mid e_{L}\right) y\right\} \\
& +\alpha\left\{\sum_{y \in \hat{Y}_{S}} P\left(y \mid e_{H}\right) C_{H, s}^{H}(y)-\sum_{y \in \hat{Y}_{P}} P\left(y \mid e_{H}\right) C_{p}^{H}(y)\right\} \\
& +(1-\alpha)\left\{\sum_{y \in \hat{Y}_{S}} P\left(y \mid e_{L}\right) C_{L, s}^{H}(y)-\sum_{y \in \hat{Y}_{P}} P\left(y \mid e_{H}\right) C_{p}^{H}(y)\right\}
\end{aligned}
$$

We can define a threshold $\alpha_{p-s}^{H}$ such that $\forall \alpha>\alpha_{p-s}^{H}$ separating is more profitable for the principal:

$$
\alpha_{p-s}^{H}=\frac{\sum_{y \in Y} P\left(y \mid e_{H}\right) y-\sum_{y \in Y} P\left(y \mid e_{L}\right) y+\sum_{y \in \hat{Y}_{S}} P\left(y \mid e_{L}\right) C_{L, s}^{H}(y)-\sum_{y \in \hat{Y}_{P}} P\left(y \mid e_{H}\right) C_{p}^{H}(y)}{\sum_{y \in Y} P\left(y \mid e_{H}\right) y-\sum_{y \in Y} P\left(y \mid e_{L}\right) y+\sum_{y \in \hat{Y}_{S}} P\left(y \mid e_{L}\right) C_{L, s}^{H}(y)-\sum_{y \in \hat{Y}_{S}} P\left(y \mid e_{H}\right) C_{H, s}^{H}(y)}
$$

We show now that $\alpha_{p-s}^{H} \geq 0$. The positivity of $\alpha_{p-s}^{H}$ is guaranteed for both the numerator and the denominator by the assumption that high efforts are always more profitable than low efforts. 
Note that if we consider $1-\alpha_{p-s}^{H}, 1-\alpha_{p-s}^{H} \geq 0 \Longleftrightarrow \sum_{y \in \hat{Y}_{P}} P\left(y \mid e_{H}\right) C_{p}^{H}(y) \geq$ $\sum_{y \in \hat{Y}_{S}} P\left(y \mid e_{H}\right) C_{H, s}^{H}(y)$

Difference in profits excluding-separating:

$\Pi_{e}\left(\hat{v}_{e}, C_{e}^{H}().\right)=\alpha \sum_{y \in Y} P\left(y \mid e_{H}\right) y-\alpha \sum_{y \in \hat{Y}_{e}^{H}} P\left(y \mid e_{H}\right) C_{e}^{H}(y)$

The difference is then:

$$
\begin{aligned}
\Pi_{P}\left(\hat{v}_{P}, C_{e}^{H}(.)\right)- & \Pi_{S}\left(\hat{v}_{S}, C_{H, s}^{H}(.), C_{L, s}^{H}(.)\right)= \\
& (1-\alpha)\left[\sum_{y \in \hat{Y}_{S}} P\left(y \mid e_{L}\right) C_{L, s}^{H}(y)-\sum_{y \in Y} P\left(y \mid e_{L}\right) y\right] \\
& +\alpha\left\{\sum_{y \in \hat{Y}_{S}} P\left(y \mid e_{H}\right) C_{H, s}^{H}(y)-\sum_{y \in \hat{Y}_{e}^{H}} P\left(y \mid e_{H}\right) C_{e}^{H}(y)\right\}
\end{aligned}
$$

$\alpha \geq \alpha_{s-e}^{H}$

$\alpha_{s-e}^{H}=\frac{\sum_{y \in Y} P\left(y \mid e_{L}\right) y-\sum_{y \in \hat{Y}_{S}} P\left(y \mid e_{L}\right) C_{L, s}^{H}(y)}{\sum_{y \in Y} P\left(y \mid e_{L}\right) y-\sum_{y \in \hat{Y}_{S}} P\left(y \mid e_{L}\right) C_{L, s}^{H}(y)+\sum_{y \in \hat{Y}_{S}} P\left(y \mid e_{H}\right) C_{H, s}^{H}(y)-\sum_{y \in \hat{Y}_{e}^{H}} P\left(y \mid e_{H}\right) C_{e}^{H}(y)}$

Proof 3: Under the rule below, which is a sufficient condition, we have:

$0 \leq \alpha_{p-s}^{o} \leq \alpha_{p-s}^{H} \leq \alpha_{s-e}^{H} \leq \alpha_{s-e}^{o} \leq 1$

1. $\sum_{y \in \hat{Y}_{s, H}} \hat{P}\left(y \mid e_{L}\right) C_{L, s}^{H}(y)-\sum_{y \in \hat{Y}_{p, H}} \hat{P}\left(y \mid e_{H}\right) C_{H, p}^{H}(y)-\sum_{y \in \hat{Y}_{s, o}} \hat{P}\left(y \mid e_{L}\right) C_{L, s}^{o}(y)+\sum_{y \in \hat{Y}_{p, o}} \hat{P}\left(y \mid e_{H}\right) C_{H, p}^{o}(y)$

$\geq \sum_{y \in \hat{Y}_{s, L}} \hat{P}\left(y \mid e_{L}\right) C_{L, s}^{H}(y)-\sum_{y \in \hat{Y}_{s, H}} \hat{P}\left(y \mid e_{H}\right) C_{H, s}^{H}(y)-\sum_{y \in \hat{Y}_{s, o}} \hat{P}\left(y \mid e_{L}\right) C_{L, s}^{o}(y)-\sum_{y \in \hat{Y}_{s, o}} \hat{P}\left(y \mid e_{H}\right) C_{H, s}^{o}(y)$

2. $\sum_{y \in Y} P\left(y \mid e_{H}\right) y-\sum_{y \in \hat{Y}_{s, H}} \hat{P}\left(y \mid e_{H}\right) C_{H, s}^{H}(y)$

$\geq \sum_{y \in Y} P\left(y \mid e_{H}\right) y-\sum_{y \in \hat{Y}_{p, H}} \hat{P}\left(y \mid e_{H}\right) C_{H, p}^{H}(y)+\sum_{y \in \hat{Y}_{s, H}} \hat{P}\left(y \mid e_{H}\right) C_{H, s}^{H}(y)-\sum_{y \in \hat{Y}_{e, H}} \hat{P}\left(y \mid e_{H}\right) C_{H, e}^{H}(y)$

3. $\sum_{y \in \hat{Y}_{s, H}} \hat{P}\left(y \mid e_{H}\right) C_{H, s}^{H}(y)-\sum_{y \in \hat{Y}_{e, H}} \hat{P}\left(y \mid e_{H}\right) C_{H, e}^{H}(y) \geq \sum_{y \in \hat{Y}_{s, o}} \hat{P}\left(y \mid e_{H}\right) C_{H, s}^{o}(y)-\sum_{y \in \hat{Y}_{e, o}} \hat{P}\left(y \mid e_{H}\right) C_{H, e}^{o}(y)$

The proof is immediate when we compare the different $\alpha$.

$\alpha_{p-s}^{H} \geq \alpha_{p-s}^{o}$

Comparing these two values is equivalent to comparing

$\left(\sum_{y \in Y} P\left(y \mid e_{H}\right) y-\sum_{y \in Y} P\left(y \mid e_{L}\right) y+\sum_{y \in \hat{Y}_{s, H}} \hat{P}\left(y \mid e_{L}\right) C_{L, s}^{H}(y)-\sum_{y \in \hat{Y}_{p, H}} \hat{P}\left(y \mid e_{H}\right) C_{p}^{H}(y)\right)$

$\mathrm{x}\left(\sum_{y \in Y} P\left(y \mid e_{H}\right) y-\sum_{y \in Y} P\left(y \mid e_{L}\right) y+\sum_{y \in \hat{Y}_{s, o}} \hat{P}\left(y \mid e_{L}\right) C_{L, s}^{o}(y)-\sum_{y \in \hat{Y}_{s, o}} \hat{P}\left(y \mid e_{H}\right) C_{H, s}^{o}(y)\right)$

and

$\left(\sum_{y \in Y} P\left(y \mid e_{H}\right) y-\sum_{y \in Y} P\left(y \mid e_{L}\right) y+\sum_{y \in \hat{Y}_{s, o}} \hat{P}\left(y \mid e_{L}\right) C_{L, s}^{o}(y)-\sum_{y \in \hat{Y}_{p, o}} \hat{P}\left(y \mid e_{H}\right) C_{H, p}^{o}(y)\right)$ 


$$
\mathrm{x}\left(\sum_{y \in Y} P\left(y \mid e_{H}\right) y-\sum_{y \in Y} P\left(y \mid e_{L}\right) y+\sum_{y \in \hat{Y}_{s, H}} \hat{P}\left(y \mid e_{L}\right) C_{L, s}^{H}(y)-\sum_{y \in \hat{Y}_{s, H}} \hat{P}\left(y \mid e_{H}\right) C_{H, s}^{H}(y)\right)
$$

A sufficient condition for the first term to be greater than the second is to assume

$$
\begin{aligned}
& \sum_{y \in \hat{Y}_{s, H}} \hat{P}\left(y \mid e_{L}\right) C_{L, s}^{H}(y)-\sum_{y \in \hat{Y}_{p, H}} \hat{P}\left(y \mid e_{H}\right) C_{H, p}^{H}(y)+\sum_{y \in \hat{Y}_{s, o}} \hat{P}\left(y \mid e_{L}\right) C_{L, s}^{o}(y)-\sum_{y \in \hat{Y}_{s, o}} \hat{P}\left(y \mid e_{H}\right) C_{H, s}^{o}(y) \\
& \geq \sum_{y \in \hat{Y}_{s, o}} \hat{P}\left(y \mid e_{L}\right) C_{L, s}^{o}(y)-\sum_{y \in \hat{Y}_{p, o}} \hat{P}\left(y \mid e_{H}\right) C_{H, p}^{o}(y)+\sum_{y \in \hat{Y}_{s, H}} \hat{P}\left(y \mid e_{L}\right) C_{L, s}^{H}(y)-\sum_{y \in \hat{Y}_{s, H}} \hat{P}\left(y \mid e_{H}\right) C_{H, s}^{H}(y)
\end{aligned}
$$

or equivalently

$$
\begin{aligned}
& \sum_{y \in \hat{Y}_{s, H}} \hat{P}\left(y \mid e_{L}\right) C_{L, s}^{H}(y)-\sum_{y \in \hat{Y}_{p, H}} \hat{P}\left(y \mid e_{H}\right) C_{H, p}^{H}(y)-\sum_{y \in \hat{Y}_{s, o}} \hat{P}\left(y \mid e_{L}\right) C_{L, s}^{o}(y)+\sum_{y \in \hat{Y}_{p, o}} \hat{P}\left(y \mid e_{H}\right) C_{H, p}^{o}(y) \\
& \geq \sum_{y \in \hat{Y}_{s, H}} \hat{P}\left(y \mid e_{L}\right) C_{L, s}^{H}(y)-\sum_{y \in \hat{Y}_{s, H}} \hat{P}\left(y \mid e_{H}\right) C_{H, s}^{H}(y)-\sum_{y \in \hat{Y}_{s, o}} \hat{P}\left(y \mid e_{L}\right) C_{L, s}^{o}(y)-\sum_{y \in \hat{Y}_{s, o}} \hat{P}\left(y \mid e_{H}\right) C_{H, s}^{o}(y)
\end{aligned}
$$

The interpretation of this assumption is that the difference of compensation between observable and hidden efforts is greater between the low compensation of the separating contract and the pooling compensation than between the low and the high compensations of the separating contract.

$$
\alpha_{s-e}^{H} \geq \alpha_{p-s}^{H}
$$

Comparing these two values is equivalent to comparing

$$
\begin{aligned}
& \left(\sum_{y \in Y} \hat{P}\left(y \mid e_{L}\right) y-\sum_{y \in \hat{Y}_{s, H}} \hat{P}\left(y \mid e_{L}\right) C_{L, s}^{H}(y)\right) \\
& \mathrm{x}\left(\sum_{y \in Y} P\left(y \mid e_{H}\right) y-\sum_{y \in Y} P\left(y \mid e_{L}\right) y+\sum_{y \in \hat{Y}_{s, H}} \hat{P}\left(y \mid e_{L}\right) C_{L, s}^{H}(y)-\sum_{y \in \hat{Y}_{s, H}} \hat{P}\left(y \mid e_{H}\right) C_{H, s}^{H}(y)\right) \\
& \text { and }
\end{aligned}
$$

$$
\begin{aligned}
& \left(\sum_{y \in Y} P\left(y \mid e_{H}\right) y-\sum_{y \in Y} P\left(y \mid e_{L}\right) y+\sum_{y \in \hat{Y}_{s, H}} \hat{P}\left(y \mid e_{L}\right) C_{L, s}^{H}(y)-\sum_{y \in \hat{Y}_{p, H}} \hat{P}\left(y \mid e_{H}\right) C_{H, p}^{H}(y)\right) \\
& \mathrm{x}\left(\sum_{y \in Y} P\left(y \mid e_{L}\right) y-\sum_{y \in \hat{Y}_{s, H}} \hat{P}\left(y \mid e_{L}\right) C_{L, s}^{H}(y)+\sum_{y \in \hat{Y}_{s, H}} \hat{P}\left(y \mid e_{H}\right) C_{H, s}^{H}(y)-\sum_{y \in \hat{Y}_{e, H}} \hat{P}\left(y \mid e_{H}\right) C_{H, e}^{H}(y)\right)
\end{aligned}
$$

As before, assuming

$$
\begin{aligned}
& \sum_{y \in Y} P\left(y \mid e_{H}\right) y-\sum_{y \in \hat{Y}_{s, H}} \hat{P}\left(y \mid e_{H}\right) C_{H, s}^{H}(y) \\
& \geq \sum_{y \in Y} P\left(y \mid e_{H}\right) y-\sum_{y \in \hat{Y}_{p, H}} \hat{P}\left(y \mid e_{H}\right) C_{H, p}^{H}(y)+\sum_{y \in \hat{Y}_{s, o}} \hat{P}\left(y \mid e_{H}\right) C_{H, s}^{H}(y)-\sum_{y \in \hat{Y}_{e, H}} \hat{P}\left(y \mid e_{H}\right) C_{H, e}^{H}(y)
\end{aligned}
$$

is a sufficient condition to have the inequality.

This assumption can be understood as requiring that the partial profits of the principal on the high-type agents for the separating contract in the hidden effort case is greater than his profits on the pooling contracts with hidden efforts plus the difference of cost between the low type in separating contract and the excluding contract in the case with hidden efforts. 
$\alpha_{s-e}^{o} \geq \alpha_{s-e}^{H}$

Comparing the $\alpha$ is equivalent to comparing

$\left(\sum_{y \in Y} P\left(y \mid e_{L}\right) y-\sum_{y \in \hat{Y}_{s, o}} \hat{P}\left(y \mid e_{L}\right) C_{L, s}^{o}(y)\right)$

$\mathrm{x}\left(\sum_{y \in Y} P\left(y \mid e_{L}\right) y-\sum_{y \in \hat{Y}_{s, H}} \hat{P}\left(y \mid e_{L}\right) C_{L, s}^{H}(y)+\sum_{y \in \hat{Y}_{s, H}} \hat{P}\left(y \mid e_{H}\right) C_{H, s}^{H}(y)-\sum_{y \in \hat{Y}_{e, H}} \hat{P}\left(y \mid e_{H}\right) C_{H, e}^{H}(y)\right)$

and

$\sum_{y \in Y} P\left(y \mid e_{L}\right) y-\sum_{y \in \hat{Y}_{s, H}} \hat{P}\left(y \mid e_{L}\right) C_{L, s}^{H}(y)$

$\mathrm{x}\left(\sum_{y \in Y} P\left(y \mid e_{L}\right) y-\sum_{y \in \hat{Y}_{s, o}} \hat{P}\left(y \mid e_{L}\right) C_{L, s}^{o}(y)+\sum_{y \in \hat{Y}_{s, o}} \hat{P}\left(y \mid e_{H}\right) C_{H, s}^{o}(y)-\sum_{y \in \hat{Y}_{e, o}} \hat{P}\left(y \mid e_{H}\right) C_{H, e}^{o}(y)\right)$.

We see first that since the constraints on the low-type separating contract are higher in the observable than in the hidden case:

$\sum_{y \in \hat{Y}_{s, H}} \hat{P}\left(y \mid e_{L}\right) C_{L, s}^{H}(y) \geq \sum_{y \in \hat{Y}_{s, o}} \hat{P}\left(y \mid e_{L}\right) C_{L, s}^{o}(y)$

and

$\sum_{y \in Y} P\left(y \mid e_{L}\right) y-\sum_{y \in \hat{Y}_{s, o}} \hat{P}\left(y \mid e_{L}\right) C_{L, s}^{o}(y) \geq \sum_{y \in Y} P\left(y \mid e_{L}\right) y-\sum_{y \in \hat{Y}_{s, H}} \hat{P}\left(y \mid e_{L}\right) C_{L, s}^{H}(y)$

We see then that assuming that

$\sum_{y \in \hat{Y}_{s, H}} \hat{P}\left(y \mid e_{H}\right) C_{H, s}^{H}(y)-\sum_{y \in \hat{Y}_{e, H}} \hat{P}\left(y \mid e_{H}\right) C_{H, e}^{H}(y) \geq \sum_{y \in \hat{Y}_{s, o}} \hat{P}\left(y \mid e_{H}\right) C_{H, s}^{o}(y)-\sum_{y \in \hat{Y}_{e, o}} \hat{P}\left(y \mid e_{H}\right) C_{H, e}^{o}(y)$

is a sufficient condition to have $\alpha_{s-e}^{o} \geq \alpha_{s-e}^{H}$

It can be seen as having the difference in cost for the high type between separating and excluding contracts greater in the hidden effort case than in the observable effort case.

Proof 4.a: If firms contract with observable efforts, awareness is necessarily maximal, and profits are entirely redistributed. The contract offered is pooling.

Observable efforts:

Bertrand competition is equivalent for the firms to maximize the agents' utilities under limited-liability constraint. This problem can be written (in a simplified way) as:

$$
\begin{aligned}
& \max _{\hat{v}, C(.)}\left\{\sum_{y \in \hat{Y}} P\left(y \mid e_{H}\right) V(C(y))-e_{H}\right\} \\
& \text { s.t. : } L L: \sum_{y \in Y} P\left(y \mid e_{H}\right)[y-C(y)] \geq 0
\end{aligned}
$$


Note that maximizing one agent's utility implies the maximization of the other, since the effort-cost is linear.

The utility-maximizing compensation respects the following condition:

$$
\frac{\partial L}{\partial C(y)}=0 \Longleftrightarrow V^{\prime}(C(y))=\lambda \text { for } y \in \hat{\mathcal{Y}}
$$

It has a corner solution for $y \in \mathcal{Y} \backslash \hat{\mathcal{Y}}$ that gives $C(y)=0$

$\lambda$ being a constant, and $V($.$) being strictly concave, since this equation is true for any$ $y$, we can conclude that $C($.$) is constant whatever y \in \hat{\mathcal{Y}}$.

The SC constraint is binding it can be written:

$$
\sum_{y \in Y} P\left(y \mid e_{H}\right) y=\sum_{y \in \hat{Y}} P\left(y \mid e_{H}\right) C(y)
$$

Since $C($.$) is a constant we obtain:$

$$
\frac{\sum_{y \in Y} P\left(y \mid e_{H}\right) y}{\sum_{y \in \hat{Y}} P\left(y \mid e_{H}\right)}=C_{F B}
$$

Considering now the optimal level of awareness, we see that it impacts both the value of $C_{F B}$ and the size of the sum in the agent's utility. The problem can be written:

$$
\max _{\hat{v}}\left\{V\left(\frac{\sum_{y \in Y} P\left(y \mid e_{H}\right) y}{\sum_{y \in \hat{Y}} P\left(y \mid e_{H}\right)}\right) \sum_{y \in \hat{Y}} P\left(y \mid e_{H}\right)\right\}
$$

The assumption $\left[x V\left(\frac{\beta}{x}\right)\right]^{\prime} \geq 0$ for any $x \in[0,1]$ and $\beta \geq 0$, which is verified by classical utility functions, like $V(x)=\frac{x^{1-\rho}}{1-\rho}$ for $x \in[0,+\infty]$ and $\left.\rho \in\right] 0,1[$ guarantees that the above function increases with the size of $\hat{Y}$.

Proof 4.b: Again, this result comes from computing the contract that maximizes the agents' utilities under limited-liability constraint of the company and incentivecompatibility constraint.

Hidden efforts: The problem becomes the following one: 


$$
\begin{aligned}
& \max _{\hat{v}, C(.)}\left\{\sum_{y \in \hat{Y}} P\left(y \mid e_{H}\right) V(C(y))-e_{H}\right\} \\
& \text { s.t. : } L L: \sum_{y \in Y} P\left(y \mid e_{H}\right)[y-C(y)] \geq 0 \\
& \text { and: } I C: \sum_{y \in \hat{Y}} \hat{P}\left(y \mid e_{H}\right) V(C(y))-e_{H} \geq \sum_{y \in \hat{Y}} \hat{P}\left(y \mid e_{L}\right) V(C(y))-e_{L}
\end{aligned}
$$

The constrained optimization problem respects the following condition:

$$
\frac{\lambda}{V^{\prime}(C(y))}=1+\frac{\gamma}{P\left(\hat{v} \mid e_{H}\right)}\left[1-\frac{\hat{P}\left(y \mid e_{L}\right)}{\hat{P}\left(y \mid e_{H}\right)}\right]
$$

In particular we have immediately $C(y)=0, \forall y \in \mathcal{Y} \backslash \hat{\mathcal{Y}}$.

Let us prove that $\lambda>0$ and $\gamma>0$ :

Suppose $\lambda=0$. Then $\sum_{y \in \hat{\mathcal{Y}}} \hat{P}\left(y \mid e_{H}\right)=\sum_{y \in \hat{\mathcal{Y}}} \hat{P}\left(y \mid e_{L}\right)=1$, and $\hat{P}\left(. \mid e_{H}\right) \neq \hat{P}\left(. \mid e_{L}\right)$, there must exist some $y \in \hat{\mathcal{Y}}$ such that $\hat{P}\left(. \mid e_{L}\right)-\hat{P}\left(. \mid e_{H}\right)<0$.

Since $\gamma \geq 0, \lambda=0 \Longrightarrow \gamma<0$, impossible. Thus, $\lambda>0$.

Suppose now $\gamma=0$. Then, $\frac{\lambda}{V^{\prime}(C(y))}=1$, which implies $C(y)=V^{\prime-1}(\lambda)$, which does not depend on $y$. Thus, $C($.$) is constant over the set of awareness of the agents, implying$ that she receives the same compensation for different outcome. Under this hypothesis, the IC constraint cannot hold.

Thus, $\gamma>0$.

Both constraints are binding. The problem can be written as below:

$$
\begin{aligned}
& \sum_{y \in Y} P\left(y \mid e_{H}\right)[y-C(y)]=0 \\
& \sum_{y \in \hat{Y}} \hat{P}\left(y \mid e_{H}\right) V(C(y))-e_{H}=\sum_{y \in \hat{Y}} \hat{P}\left(y \mid e_{L}\right) V(C(y))-e_{L}
\end{aligned}
$$

Let us answer now the second question: Are the agents made fully aware?

Following the notations of Auster (2013) let us denote $E_{\Upsilon_{A} \cup v}$ and $C_{\Upsilon_{A} \cup v}$ respectively the expectation operator and the optimal compensation under awareness $\Upsilon_{A} \cup v$. Consider the binding IC constraint under the agents' initial level of awareness:

$$
E_{\Upsilon_{A}}\left(V\left(C_{\Upsilon_{A}}(y)\right) \mid e_{H}\right)-e_{H}=E_{\Upsilon_{A}}\left(V\left(C_{\Upsilon_{A}}(y)\right) \mid e_{L}\right)-e_{L}
$$

In a generic way we have 
$P\left(v=0 \mid e_{H}\right)=1-\epsilon, P\left(v=1 \mid e_{H}\right)=\epsilon, P\left(v=0 \mid e_{L}\right)=1-\epsilon^{\prime}, P\left(v=1 \mid e_{L}\right)=\epsilon^{\prime}$ with $\epsilon, \epsilon^{\prime} \in[0,1]$

Increasing awareness by one contingency, but keeping the same compensation scheme in a first step gives us the following relation:

$$
\begin{aligned}
& E_{\Upsilon_{A} U v}\left(V\left(C_{\Upsilon_{A}}(y)\right) \mid e_{H}, v=0\right)-\epsilon\left\{E_{\Upsilon_{A} U v}\left(V\left(C_{\Upsilon_{A}}(y)\right) \mid e_{H}, v=0\right)-E_{\Upsilon_{A} U v}\left(V\left(C_{\Upsilon_{A}}(y)\right) \mid e_{H}, v=1\right)\right\}-e_{H} \\
& \gtreqless E_{\Upsilon_{A} U v}\left(V\left(C_{\Upsilon_{A}}(y)\right) \mid e_{L}, v=0\right)-\epsilon^{\prime}\left\{E_{\Upsilon_{A} U v}\left(V\left(C_{\Upsilon_{A}}(y)\right) \mid e_{L}, v=0\right)-E_{\Upsilon_{A} U v}\left(V\left(C_{\Upsilon_{A}}(y)\right) \mid e_{L}, v=1\right)\right\}-e_{L}
\end{aligned}
$$

Adding this contingency allows for more slack iff

$$
\begin{aligned}
& \epsilon\left\{E_{\Upsilon_{A} U v}\left(V\left(C_{\Upsilon_{A}}(y)\right) \mid e_{H}, v=0\right)-E_{\Upsilon_{A} U v}\left(V\left(C_{\Upsilon_{A}}(y)\right) \mid e_{H}, v=1\right)\right\} \\
& >\epsilon^{\prime}\left\{E_{\Upsilon_{A} U v}\left(V\left(C_{\Upsilon_{A}}(y)\right) \mid e_{L}, v=1\right)-E_{\Upsilon_{A} U v}\left(V\left(C_{\Upsilon_{A}}(y)\right) \mid e_{L}, v=0\right)\right\}
\end{aligned}
$$

- For fixed differences on both sides of the inequality, the SP has more interest to add the contingency for a bigger relative impact of exerting a high effort.

- For a fixed difference in probabilities conditionally on the effort, the slack results from the relatively lower difference of what a $v=1$ adds compared to a 0 one, conditionally on the effort.

This problem leads to partial awareness in a hidden effort contract. 